\title{
Optimization of Prognostication Model about the Spread of Ebola Based on SIR Model
}

\author{
Xueer Bai \\ North China Electric Power University, Baoding 071000, China \\ 523630491@qq.com
}

Keyword: SEIR Model, SEIQRD Model, Ebola forecasting

\begin{abstract}
Aiming to help eradicate the Ebola outbreak in West Africa, the article optimizes the classical SIR model which works on predicting the spread of infectious diseases, providing insight into not only forecasting how the disease continues to develop but also what measurement should be taken. The models can be applied to forecast the spread of other severe infectious diseases that can cause quantities of death in a short time.
\end{abstract}

\section{Introduction}

SIR Model is a classic model that can effectively predict the spread of disease. In SIR model, the population are divided into three sections--S(t)(Suspected), $I(t)($ Infectious), $R(t)($ Removed $) . S(t)$ is the amount of people who are likely to become infected, $I(t)$ is the amount of infectious people, and $R(t)$ is the amount of people considered to be recovered or dead.

\section{Model building and analysis}

\section{Model Building}

Prediction of free-spread epidemic

Since people infected with the Ebola virus needs to go through an incubation period of 2 to 21 days before the onset of symptoms and transmission of infection, the author has established a SEIR Model with a new group of people added----E(t)(Exposed). $E(t)$ is the amount of people incubating the infection. Here we neglect natural birth rate, mortality rate and migration of the population, thus taking the total population $\mathrm{N}$ as a constant. Assume:

t: the day that Ebola has spread;

$\mathrm{N}$ : the population of the country;

$\beta$ : the effective contact rate;

$\frac{1}{\sigma}:$ the average days of incubation period;

$\sigma:$ the proportion of people who is from Exposed to Infectious;

$\delta$ : the isolated rate;

$\gamma$ : the lethality rate;

$\varepsilon$ : the proportion of people who is from Quarantine to Dead;

$\rho:$ the cure rate ;

$\alpha$ : the rate of vaccines work.

Therefore we can deduce that at $t$ moment:

The probability of $\mathrm{S}(\mathrm{t})$ becoming $\mathrm{E}(\mathrm{t})$ is $\frac{\beta I(t)}{N}$, that is, the change rate of $\mathrm{S}(\mathrm{t})$ is $-\frac{\beta S(t) I(t)}{N}$. With $\sigma E(t)$ turning into $\mathrm{I}(\mathrm{t})$, the change rate of $\mathrm{E}(\mathrm{t})$ is $\frac{\beta S(t) I(t)}{N}-\sigma E(t)$. And since at $\mathrm{t}$ moment there will be $\gamma I(t)$ becoming $\mathrm{R}(\mathrm{t})$, the change rate of $\mathrm{I}(\mathrm{t})$ is $\sigma E(t)-\gamma I(t)$ while the change rate of $\mathrm{R}(\mathrm{t})$ is $\gamma I(t)$. The governing equations describing the evolution and dynamics of SEIR model can be described as follows: 


$$
\begin{gathered}
\frac{\mathrm{dS}(\mathrm{t})}{\mathrm{dt}}=-\frac{\beta S(t) I(t)}{N} \\
\frac{\mathrm{dE}(\mathrm{t})}{\mathrm{dt}}=\frac{\beta S(t) I(t)}{N}-\sigma E(t) \\
\frac{\mathrm{dI}(\mathrm{t})}{\mathrm{dt}}=\sigma E(t)-\gamma I(t) \\
\frac{\mathrm{dR}(\mathrm{t})}{\mathrm{dt}}=\gamma I(t)
\end{gathered}
$$

\section{Prediction of epidemic with medication and quarantine}

As the disease progresses, the Government will take isolation and medication measures to curb the spread of the virus, so an improved model based on the original one is established as SEIQRD Model. In this new model, people are divided into six sections--S(t)(Suspected), E(t)(Exposed), $I(t)$ (Infected), $Q(t)($ Quarantined), $R(t)$ (Recovered) and $D(t)($ Dead). What's more, the following parameters are added into the new model: $\delta, \gamma, \varepsilon, \rho, \alpha$.

Then we can get new ordinary differential equations as follows:

$$
\begin{gathered}
\frac{\mathrm{dS}(\mathrm{t})}{\mathrm{dt}}=-\frac{\beta}{N}(1-\alpha) S(t)(1-\delta-\gamma-\rho) I(t) \\
\frac{\mathrm{dE}(\mathrm{t})}{\mathrm{dt}}=\frac{\beta}{N}(1-\alpha) S(t)(1-\delta-\gamma-\rho) I(t)-\sigma E(t) \\
\frac{\mathrm{dI}(\mathrm{t})}{\mathrm{dt}}=\sigma E(t)-(\delta+\gamma+\rho) I(t) \\
\frac{\mathrm{dQ}(\mathrm{t})}{\mathrm{dt}}=\delta I(t)-(\varepsilon+\rho) Q(t) \\
\frac{\mathrm{dR}(\mathrm{t})}{\mathrm{dt}}=\rho(I(t)+Q(t)) \\
\frac{d D(t)}{d(t)}=\gamma I(t)+\varepsilon Q(t)
\end{gathered}
$$

Here are some special instructions about $\alpha: \alpha=\alpha_{1} \times \alpha_{2} \times \alpha_{3}$

$\alpha_{1}$ is the ratio of the amount of drugs to the amount of patients. $\alpha_{2}$ is the proportion of total amount of drugs that the amount of drugs demanded by each country accounts for. $\alpha_{3}$ is the effective cure rate of each patient. Apparently, $\alpha$ signifies the degree of drugs or vaccines controlling the disease. That is to say, the spread of the disease will be contained in different degrees as $\alpha$ values differently.

\section{Model Solving}

The article picked up two countries where the outbreak is the most severe---Sierra Leone and Liberia as the research objects. According to the data from the WHO official website, the article chose the data on December 31th, 2014 as initial values.

\section{SEIR Model solving}

Here we take the parameters as follows: $\frac{1}{\sigma}=10$ days, $\beta$ (Liberia) $=0.237, \beta$ (Sierra Leone $)=0.106$. We've worked out the ordinary differential equations and drawn change curves as follows: 


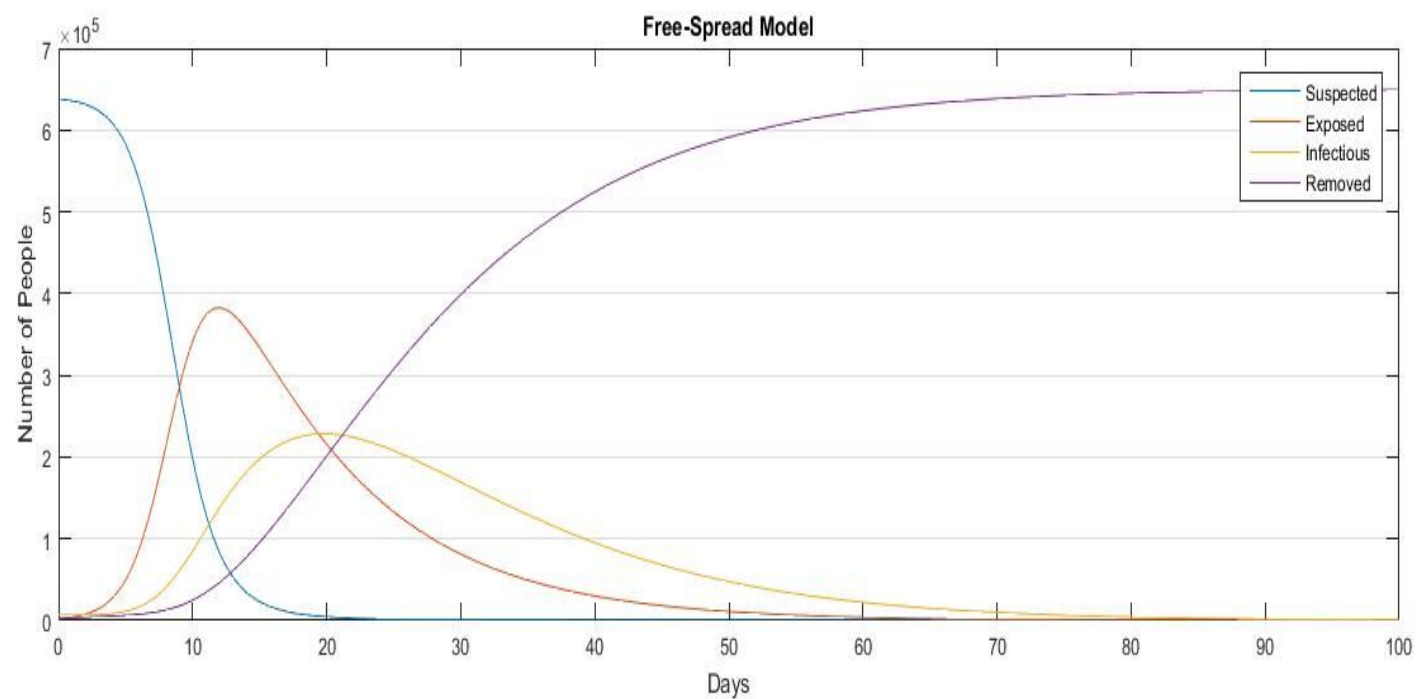

Figure 1: SEIR model of Ebola in Sierra Leone

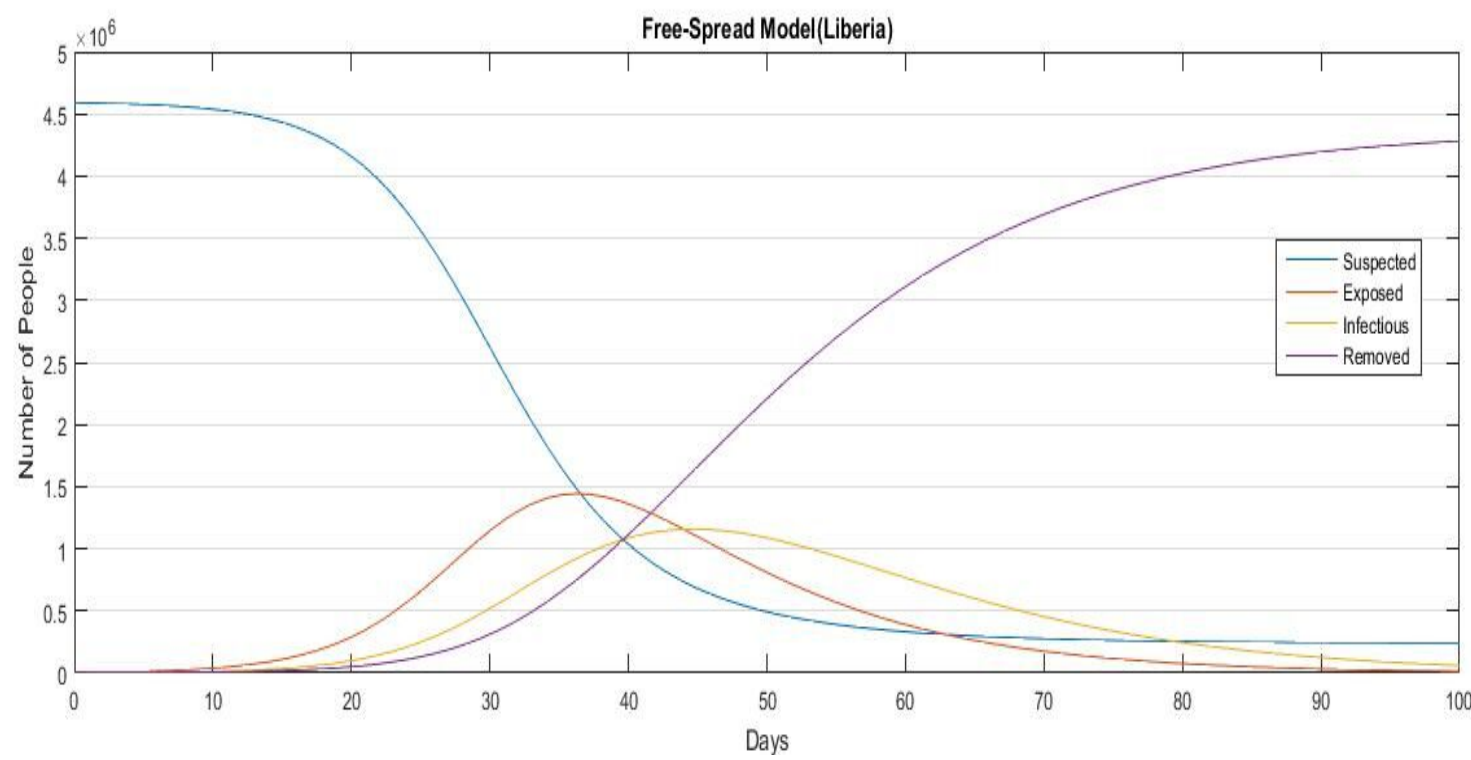

Figure 2: SEIR model of Ebola in Liberia

\section{SEIQRD Model solving}

According to the data we have got, we take the parameters as follows: $\rho=0.15, \delta=0.197$, $\frac{1}{\sigma}=10$ days,$\frac{1}{\gamma}=10.38$ days,$\varepsilon=0.75 ; \beta($ Liberia $)=0.674, \alpha($ Liberia $)=0.0173 ; \beta($ Sierra Leone $)=$ $0.117, \alpha($ Sierra Leone $)=0.1142$.

Thus the ordinary differential equations and change curves are shown in Figure 3 and Figure 4.

\section{Summary}

Comparing Figure 3.4 to Figure 1.2, it is easy to conclude that after taking medication and quarantine, a marked reduction in the amounts of the Exposed and the Infected occurs, especially the Exposed. Besides, considering that the probability of self-cure is quite small without medication or quarantine, the Removed section in SEIR Model, which is made up of the Recovered and the Dead, mainly concludes the Dead. Therefore comparing the curves of $\mathrm{R}(\mathrm{t})$ in Figure1.2.3 with the curves of $\mathrm{D}(\mathrm{t})$ in Figure 4.5.6, we can find that the amount of the Dead declines while the amount of the Recovered increases. Furthermore, the timelier isolation and the more sufficient medication supplies are, the lower infectious rate will be. To conclude, medication or vaccine treatments and quarantine are effective ways to contain the epidemic. 


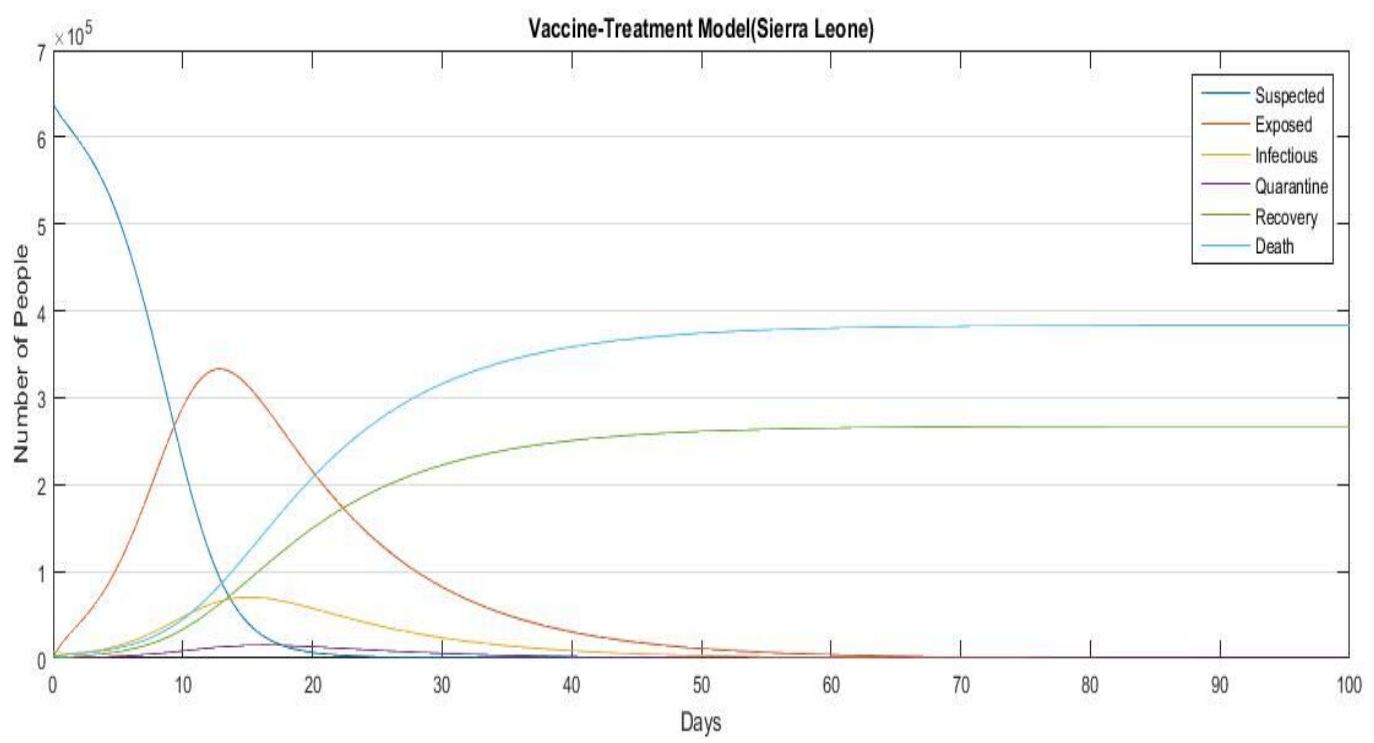

Figure 3: SEIQRD model of Ebola in Sierra Leone

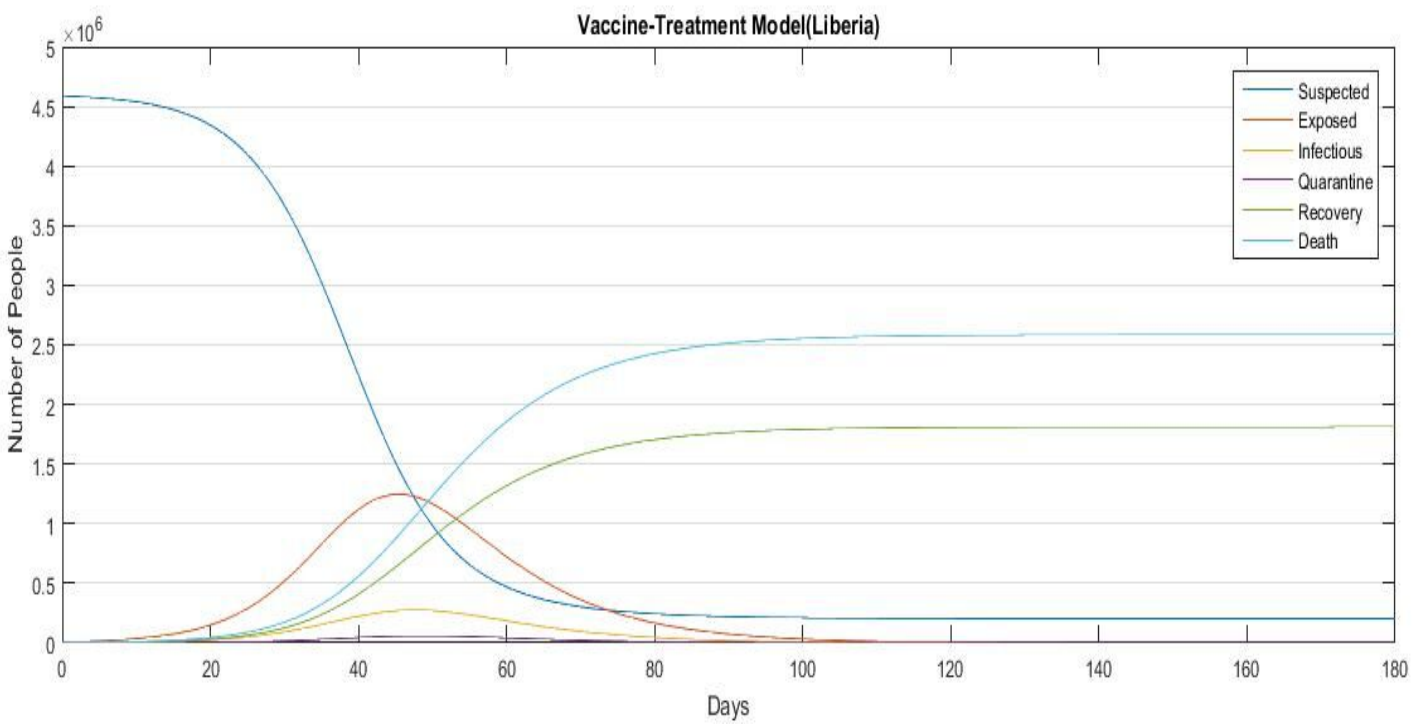

Figure 4: SEIQRD model of Ebola in Liberia

\section{References}

[1] HE Wenjun, YU Bingqing, GUO Wenmin, WANG Juping, GUO Dongxing, Prediction of the Development of the Ebola and the Research on the Optimal Drug Delivery System[J]. Journal of Shanxi Medical University, 2015, (08):783-787

[2] [C], 2015 (Fourth) National Undergraduate Thesis Statistical Modeling Contest, Beijing: China Statistical Society Education, 2015:191-217

[3] XU ZhiJing, XU Zhankai, LIU Lie, ZU Zhenghu, ZHENG Tao, Review of Researches on the Projection of Ebola Dynamics in Western Africa [J]. Letters in Biotechnology, 2015, (01):22-32 